\title{
Oral supplementation with ursolic acid ameliorates sepsis-induced acute kidney injury in a mouse model by inhibiting oxidative stress and inflammatory responses
}

\author{
ZHENYU ZHANG $^{1}$, HONG ZHANG ${ }^{2}$, RUI $\mathrm{CHEN}^{1}$ and ZHONG WANG ${ }^{1}$ \\ ${ }^{1}$ Department of Critical Care Medicine, Beijing Tsinghua Changgung Hospital, Beijing 102218; \\ ${ }^{2}$ Department of Applied Chemistry, Peking University, Beijing 100871, P.R. China
}

Received August 27, 2016; Accepted August 16, 2017

DOI: $10.3892 / \mathrm{mmr} .2018 .8767$

\begin{abstract}
Ursolic acid (UA) as a multiple bioactive native compound has recently been demonstrated to treat sepsis in animal models. However, the beneficial effects of UA in sepsis-induced acute kidney injury (AKI) are not completely understood. In the present study, the effect of UA on sepsis-induced AKI in cecal ligation and puncture (CLP) surgery mice was investigated. Renal histomorphological analysis was performed by hematoxylin and eosin staining. The expression of inflammatory markers in the kidney of septic mice was measured by reverse transcription-quantitative polymerase chain reaction and western blotting. The results demonstrated that UA administration improved survival in septic mice induced by CLP surgery. The treatment with UA revealed protection against AKI induced by CLP surgery, including the alleviation of glomerular damage and vacuolization in the proximal tubules. In addition, the effects of UA on oxidative stress and inflammation in septic mice were determined. The findings suggested that UA may protect against sepsis-induced AKI by inhibiting reactive oxygen species and inflammatory cytokines, including tumor necrosis factor- $\alpha$, interleukin (IL)- $1 \beta$ and IL- 6 , in the kidney from septic mice. Finally, UA inhibited CLP-induced activation of nuclear factor- $\kappa \mathrm{B}$ signaling in the kidney from septic mice. The findings of the present study demonstrated that UA may be used as a potential therapeutic agent for complications of sepsis, especially for sepsis-induced AKI.
\end{abstract}

\section{Introduction}

Acute kidney injury (AKI) is a common problem in critically ill patients, particularly in the intensive care unit (ICU) (1). Sepsis

Correspondence to: Dr Zhong Wang, Department of Critical Care Medicine, Beijing Tsinghua Changgung Hospital, 168 Litang Road, Beijing 102218, P.R. China

E-mail: zhong_wang1@163.com

Key words: ursolic acid, sepsis, acute kidney injury, oxidative stress, inflammatory response and septic shock are factors that contribute to the development of AKI, and $\geq 50 \%$ mortality of sepsis patients in the ICU is associated with AKI (2). Evidence suggests that patients with non-severe pneumonia with sepsis have a significantly higher incidence of AKI and increased inflammatory responses $(3,4)$. However, the underlying mechanisms are not completely understood. A growing body of evidence has suggested that sepsis-induced oxidative stress and inflammatory responses contributed to sepsis-associated organ dysfunction, including AKI (5-7).

Ursolic acid (UA) as a pentacyclic triterpenoid compound is isolated from many plants, including Ligustrum lucidum, Arctostaphylos uva-ursi and Eriobotrya japonica, which are widely used as Chinese herbal medicines (8). UA is readily absorbed from daily foods, such as Fructus Crataegi (9), Fructus Chaenomelis Lagenariae (10) and Fructus Mume (11). It is generally known that UA exhibits multiple biological activities, such as anti-inflammatory (12), anti-oxidant (13) and anti-tumor properties (14). Emerging evidence has demonstrated that UA has a beneficial effect on hyperglycemia-induced renal injury (15). In addition, UA ameliorates carbon tetrachloride-induced oxidative DNA damage and inflammation in kidneys of rats (16). Previous research has shown that UA exerts protective effects on sepsis-induced organ damage $(17,18)$. However, the beneficial effect and underlying molecular mechanisms of UA in sepsis-induced AKI are not clearly delineated. As sepsis is an acute inflammatory and oxidative stress disorder involved in AKI, inhibiting excessive inflammatory responses and oxidative stress may be an effective therapeutic target.

In the present study, it was hypothesized that UA may ameliorate sepsis-induced AKI via the inhibition of oxidative stress and inflammatory responses. This study may further increase understanding of organ failure prevention and the underlying mechanisms of UA, which may be useful in managing sepsis-induced AKI.

\section{Materials and methods}

Experimental animals. Ten-week-old male imprinting control region mice $(n=64)$ were purchased from Charles River Laboratories, Inc., (Wilmington, MA, USA) and were allowed to acclimate to the environment for 1 week. The mice were fed 
under controlled conditions: Temperature $\left(25 \pm 2^{\circ} \mathrm{C}\right)$, humidity $(55 \pm 5 \%)$ and 12 -h light/dark cycle, and the mice were given free access to food and tap water.

Sepsis was induced in mice undergoing cecal ligation and puncture (CLP) surgery (CLP Group, $n=8$ ). Mice were anesthetized with $2 \%$ pentobarbital, and a $1-$ to $2-\mathrm{cm}$ midline incision was made along the linea-alba of the abdominal muscle to isolate and exteriorize the cecum. A total of $75 \%$ of the cecum was ligated with a 4-0 silk suture, and the cecum was punctured twice with a 21-gauge needle. A small amount (1 droplet) of feces was gently extruded from the holes to ensure patency. The cecum was then returned to the peritoneal cavity and the abdominal incision was closed with 4-0 silk sutures. After the operation, $1 \mathrm{ml}$ pre-warmed normal saline was administered into the peritoneal cavity. The survival rate was examined over the whole experiment (up to 4 days). For the UA (Aldrich Chemical Co., Milwaukee, WI, USA; high performance liquid chromatography, $98 \%$ ) treatment groups $(2 \mathrm{mg} / \mathrm{kg}$, low-concentration, $\mathrm{n}=8$ or $20 \mathrm{mg} / \mathrm{kg}$, high-concentration, $\mathrm{n}=8$ ), mice received intraperitoneal administration of UA 2 weeks before CLP surgery. In the sham-operated group (control group, $n=8$ ), mice underwent the same procedure but were neither ligated nor punctured, and the mice were intraperitoneally injected with $2 \mathrm{ml}$ PBS. In another experiment, the $96 \mathrm{~h}$ survival of CLP mice with or without UA treatment was observed ( $\mathrm{n}=8$ in each group).

The animal study was approved by the Animal Care and Use Committee at the Beijing Tsinghua Changgung Hospital (Beijing, China; permit number: TCH-2015-0019).

Serum inflammatory cytokine measurement. The effects of UA on serum cytokine levels were analyzed by ELISA at $24 \mathrm{~h}$ after CLP, which was determined to be the optimum measuring time in previous studies $(17,19)$. Serum levels of tumor necrosis factor- $\alpha$ (TNF- $\alpha$; cat. no. E-EL-M0049), interleukin (IL)-1 $\beta$ (cat. no. E-EL-M0037) and IL-6 (cat. no. E-EL-M0044) were detected using a mouse bioactive ELISA assay, according to the manufacturer's protocol (Elabscience Biotechnology Co., Ltd., Wuhan, China), there were three replicates for each sample tested, $\mathrm{n}=8$ in the control group; $\mathrm{n}=6$ in CLP group ( 2 mice succumbed within $24 \mathrm{~h}$ ); $\mathrm{n}=7$ in $\mathrm{UA}(\mathrm{L})+\mathrm{CLP}$ group (1 mouse succumbed within $24 \mathrm{~h}$ ); $\mathrm{n}=8$ in $\mathrm{UA}(\mathrm{H})+\mathrm{CLP}$ group.

Measurement of serum creatinine (Scr) and blood urea nitrogen $(B U N)$. Blood samples were collected at $24 \mathrm{~h}$ after CLP surgery in each group and renal function was monitored by measuring the concentration of BUN (cat. no. C013-2) and Scr (cat. no. C011-1) in serum using an enzymatic kinetic methods and picric acid methods detection kits (Nanjing Jiancheng Bioengineering Institute, Nanjing, China), according to the manufacturer's protocol. For BUN and Scr, $n=8$ in the control group; $\mathrm{n}=6$ in CLP group ( 2 mice succumbed within $24 \mathrm{~h}$ ); $\mathrm{n}=7$ in $\mathrm{UA}(\mathrm{L})+\mathrm{CLP}$ group (1 mouse succumbed within $24 \mathrm{~h}) ; \mathrm{n}=8$ in $\mathrm{UA}(\mathrm{H})+\mathrm{CLP}$ group.

Measurement of reactive oxygen species (ROS). The renal tissues were harvested and homogenized in phosphate buffer at $24 \mathrm{~h}$ after CLP surgery in each group. ROS (ID: GMS10016.3; Genmed Scientifics, Inc., Shanghai, China) levels were measured using a dichloro-dihydro-fluorescein diacetate (DCFH-DA) assay, according to the manufacturer's protocol. In brief, kidney tissue whole homogenate $(100 \mu \mathrm{l})$ was added to $1.4 \mathrm{ml} 50 \mathrm{mM}$ phosphate buffer in dark-adapted counting vials. After dark adaptation for $1 \mathrm{~h}$ at room temperature, the homogenate was incubated with $10 \mu \mathrm{M}$ DCFH-DA for $20 \mathrm{~min}$ at $37^{\circ} \mathrm{C}$ in the dark for the detection of ROS. The samples were counted every $20 \mathrm{sec}$ for 3 min using a luminometer (Autolumat Plus LB953; Berthold Technologies GmbH, Bad Wildbad, Germany).

Measurement of 8-hydroxy-2'-deoxyguanosine (8-OHdG). Plasma levels of an oxidative stress marker, 8-OHdG, was measured using a DNA damage ELISA kit (cat. no. ADI-EKS-350; Enzo Life Sciences, Inc., Farmingdale, NY, USA), according to the manufacturer's protocol.

Hematoxylin and eosin staining. Formalin-fixed (4\% formalin at room temperature for $24 \mathrm{~h}$ ) and paraffin-embedded kidney tissues were cut into $\sim 5 \mu \mathrm{m}$-thick sections, which were stained with hematoxylin and eosin (cat. no. C0105; Beyotime Institute of Biotechnology, Haimen, China) at room temperature for 1-2 min, and visualized under a light microscope (model DM 2500; Leica Microsystems, Inc., Buffalo Grove, IL, USA).

Reverse transcription-quantitative polymerase chain reaction $(R T-q P C R)$. Total RNA was extracted from mouse kidney [ $\mathrm{n}=8$ in the control group; $\mathrm{n}=6$ in CLP group ( 2 mice succumbed within $24 \mathrm{~h}$ ); $\mathrm{n}=7$ in UA(L)+CLP group (1 mouse succumbed within $24 \mathrm{~h}$ ); $\mathrm{n}=8$ in UA (H) +CLP group] using TRIzol (Invitrogen; Thermo Fisher Scientific, Inc., Waltham, MA, USA) and reverse transcribed into cDNA using a SuperScriptIII reverse transcriptase kit (Invitrogen; Thermo Fisher Scientific, Inc.), following the manufacturer's protocol. RT-qPCR was used to determine mRNA expression levels using SYBR-Green Master mix (Invitrogen; Thermo Fisher Scientific, Inc.) on a Stratagene MX3005P system (Agilent Technologies, Inc., Santa Clara, CA, USA). The thermocycling conditions were as follows: $95^{\circ} \mathrm{C}$ for 10 min followed by 40 cycles of $95^{\circ} \mathrm{C}$ for $15 \mathrm{sec}, 60^{\circ} \mathrm{C}$ for $30 \mathrm{sec}$ and $72^{\circ} \mathrm{C}$ for $30 \mathrm{sec}$. GAPDH served as an internal standard. Relative gene expression was calculated using the $2^{-\Delta \Delta C q}$ method (20). The following primers were used: Forward, 5'-CACCACCATCAAGGACTCAA-3' and reverse, 5'-GAG ACAGAGGCAACCTGACC-3' for TNF- $\alpha$; forward, 5'-TCG CCAGTGAAATGATGGCTTA-3' and reverse, 5'-GTCCAT GGCCACAACAACTGA-3' for IL-1 $\beta$; forward, 5'-CGGAGA GGAGACTTCACAGAG-3' and reverse, 5'-CATTTCCACGAT TTCCCAGA-3' for IL-6; forward, 5'-GGGTGAGAACGGGCC TGGAGGA-3' and reverse, 5'-GTAGAAGCCCGGTGTGCC GTGA-3' for superoxide dismutase (SOD)1; forward, 5'-TCG GTAACGTGAGTGTGGCAAT-3' and reverse, 5'-CGTGTT GGACCGGTGTGCACCGT-3' for SOD2; forward, 5'-ACA GGGGAGGTGATAGCATT-3' and reverse, 5'-GACCAAAAG CCTTCATACATCTC-3' for GAPDH. For PCR, $n=8$ in the control group; $n=6$ in CLP group (2 mice death within $24 \mathrm{~h}$ ); $\mathrm{n}=7$ in $\mathrm{UA}(\mathrm{L})+\mathrm{CLP}$ group ( 1 mouse death within $24 \mathrm{~h}$ ); $\mathrm{n}=8$ in $\mathrm{UA}(\mathrm{H})+\mathrm{CLP}$ group. The experiment was repeated twice. Three replicates were performed for each sample.

Western blotting. Kidneys were homogenized and lysed in NP-40 buffer (Beyotime Institute of Biotechnology, Haimen, China). Following 5-10 min boiling, homogenates were centrifuged at $10,000 \times \mathrm{g}$ at $4^{\circ} \mathrm{C}$ for $10 \mathrm{~min}$ to obtain the supernatant. 
Protein samples $(50 \mu \mathrm{g})$ were separated by $10 \%$ SDS-PAGE and transferred to polyvinylidene difluoride membranes (EMD Millipore, Billerica, MA, USA). Membranes were blocked with $5 \%(\mathrm{w} / \mathrm{v})$ non-fat milk powder in Tris-buffered saline containing $0.1 \%(\mathrm{w} / \mathrm{v})$ Tween 20 for $2 \mathrm{~h}$ at room temperature, and subsequently incubated with the following primary antibodies: TNF- $\alpha$ (cat. no. sc-52746), IL-1 $\beta$ (cat. no. sc-515598), IL-6 (cat. no. sc-32296), NF-кB (cat. no. sc-114) and $\beta$-actin (cat. no. sc-517582; all from Santa Cruz Biotechnology, Inc., Dallas, TX, USA; all 1: 1,000 ), at $4^{\circ} \mathrm{C}$ overnight. After being washed, the membranes were incubated with horseradish peroxidase-conjugated anti IgG (cat. no. sc-516102; 1:10,000; Santa Cruz Biotechnology, Inc.) at room temperature for $2 \mathrm{~h}$. Signal detection was carried out with an enhanced chemiluminescence system (GE Healthcare, Chicago, IL, USA), and protein bands were analyzed with Quantity One ${ }^{\circledR}$ software version 4.5 (Bio-Rad Laboratories, Inc., Hercules, CA, USA). For western blotting, $n=8$ in the control group; $n=6$ in CLP group ( 2 mice succumbed within $24 \mathrm{~h}$ ); $\mathrm{n}=7$ in $\mathrm{UA}(\mathrm{L})+\mathrm{CLP}$ group (1 mouse succumbed within $24 \mathrm{~h}$ ); $\mathrm{n}=8$ in $\mathrm{UA}(\mathrm{H})+\mathrm{CLP}$ group.

Statistical analysis. Data are presented as the mean \pm standard deviation for each group. Statistical analyses were performed by using GraphPad Prism version 6.0 software (GraphPad Software, Inc., La Jolla, CA, USA). Inter-group differences were analyzed by one-way analysis of variance followed by Tukey's multiple comparison post-hoc test. $\mathrm{P}<0.05$ was used to indicate a statistically significant difference.

\section{Results}

UA administration improves survival in septic mice. A previous study demonstrated that UA administration improves survival in CLP-induced lung injury septic mice (17). In line with these findings, the present study revealed that UA pretreatment before the induction of sepsis by CLP significantly improved the survival of mice undergoing CLP compared with mice treated with CLP alone. Approximately $90 \%$ of mice in the CLP group died at $72 \mathrm{~h}$ after undergoing CLP surgery. Administration of UA $(20 \mathrm{mg} / \mathrm{kg})$ had a protective effect against lethality induced by CLP $(\mathrm{P}<0.05)$, and increased the animal survival rate from 12.5 to $50 \%$. However, a low concentration of UA $(2 \mathrm{mg} / \mathrm{kg})$ did not significantly improve the survival of CLP-induced septic mice (Fig. 1).

UA improves sepsis-induced AKI in mice. Previous studies have suggested that UA alleviates sepsis-induced acute lung injury in rodent models $(17,18)$. However, there appears to be no associated reports on sepsis-induced AKI. To determine the role of UA in sepsis-induced AKI in mice, renal histological examination by hematoxylin and eosin staining was implemented. In control mice, relatively intact structures of the kidney tissues were observed. By contrast, glomerular damage and vacuolization were observed in the proximal tubules in the CLP group. Low concentrations of UA $(2 \mathrm{mg} / \mathrm{kg})$ had no dramatic improvement in renal tissue damage in septic mice. However, high concentrations of UA $(20 \mathrm{mg} / \mathrm{kg})$ ameliorated the renal tissue damage in septic mice (Fig. 2A). It is well known that AKI is a serious complication in septic rats, and BUN and Scr are frequently used as biomarkers in the

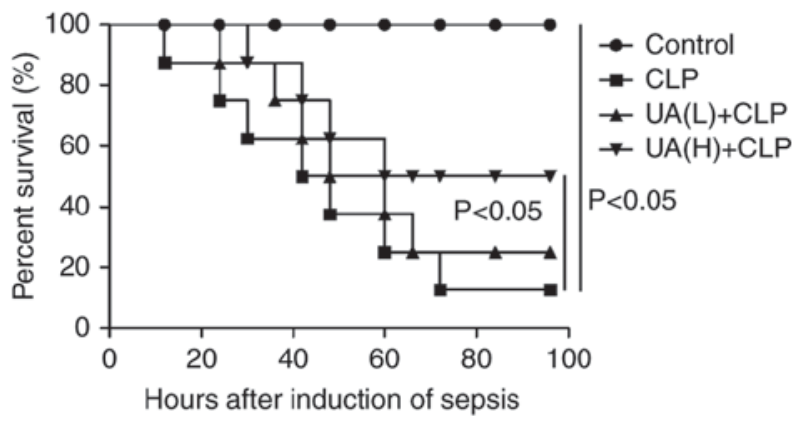

Figure 1. UA treatment ameliorates survival of mice subjected to CLP. Low concentration $(2 \mathrm{mg} / \mathrm{kg})$ or high concentration $(20 \mathrm{mg} / \mathrm{kg})$ UA was administered to mice 2 weeks before the induction of sepsis by CLP. UA, ursolic acid; CLP, cecal ligation and puncture.

early stages of the development of AKI (21). In the present study, BUN and Scr levels were measured at $24 \mathrm{~h}$ after CLP surgery to evaluate renal function in septic mice. As shown in Fig. 2B and C, BUN and Scr were significantly increased in mice undergoing CLP surgery compared with the control group. Administration of high concentrations of UA significantly reversed the CLP-induced upregulation of BUN and Scr in septic mice $(\mathrm{P}<0.05)$. Low concentrations of UA did not significantly reduce the levels of BUN and Scr.

$U A$ restrains $C L P$-induced oxidative stress damage in septic mice. The effect of UA on CLP-induced ROS in the kidney of septic mice was measured. Treatment with CLP surgery resulted in a significant increase in ROS production compared with the control group $(\mathrm{P}<0.001)$, whereas pretreatment with high concentrations of UA significantly inhibited CLP-induced ROS production ( $\mathrm{P}<0.05$; Fig. $3 \mathrm{~A})$. In addition, plasma 8-OHdG levels, a measure of DNA oxidative damage, were significantly higher in the CLP group compared with the control group $(\mathrm{P}<0.001)$, whereas pretreatment with high concentrations of UA significantly reversed CLP-induced 8-OhdG ( $\mathrm{P}<0.05$; Fig. 3B). Furthermore, SOD1 and SOD2, as antioxidant enzymes, serve crucial roles in the defense against oxygen-free radicals (22). In the present study, the mRNA expression levels of SOD1 and SOD2 were measured by RT-qPCR in mouse kidney tissue, and the results demonstrated that SOD1 and SOD2 were significantly decreased in the kidney from CLP septic mice compared with that from control animals $(\mathrm{P}<0.01)$. High concentrations of UA significantly reversed the CLP-induced downregulation of SOD1 and SOD2 in CLP septic mice ( $\mathrm{P}<0.05$; Fig. $3 \mathrm{C}$ and $\mathrm{D})$.

UA suppresses inflammatory responses in septic mice. Previous studies have suggested that oxidative stress directly or indirectly elevates inflammatory responses $(21,23,24)$. Therefore, serum TNF- $\alpha$, IL-1 $\beta$ and IL-6 levels were measured at $24 \mathrm{~h}$ after CLP surgery. Serum TNF- $\alpha$ (Fig. 4A), IL-1 $\beta$ (Fig. 4B) and IL-6 (Fig. 4C) levels were significantly increased in the CLP group compared with the control group. However, treatment with high concentrations of UA significantly decreased the levels of these inflammatory cytokines in septic mice $(\mathrm{P}<0.05)$. In addition, the mRNA expression levels of TNF- $\alpha$ (Fig. 4D), IL-1 $\beta$ (Fig. 4E) and IL-6 (Fig. 4F) were measured using RT-qPCR in kidney tissues, and the results 

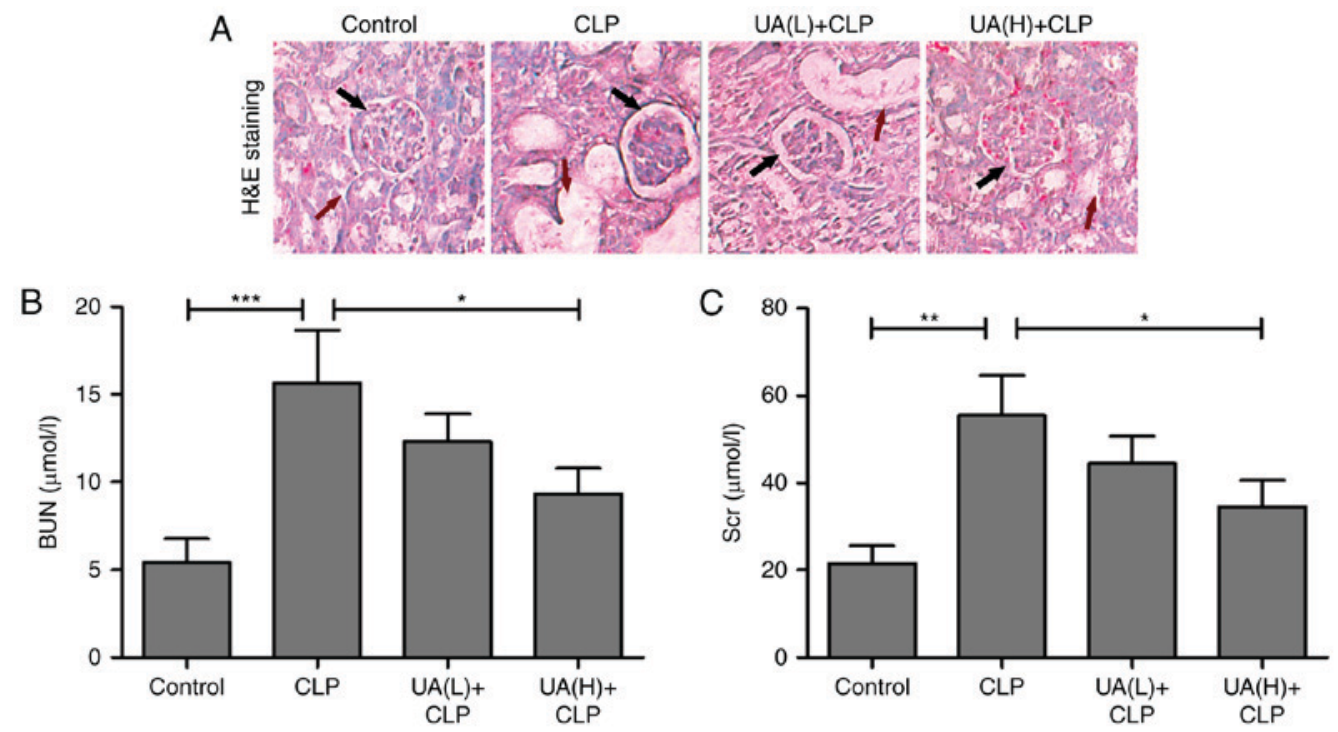

Figure 2. UA improves sepsis-induced AKI. (A) Hematoxylin and eosin staining of kidney tissue from a representative mouse in the control, CLP, UA(L)+CLP and $\mathrm{UA}(\mathrm{H})+\mathrm{CLP}$ groups. Magnification, x100. Black arrows indicated glomerular damage degree, and red arrows indicated the degree of vacuolization in the proximal tubules. Concentration of (B) BUN and (C) Scr in serum of mice of each group. UA, ursolic acid; CLP, cecal ligation and puncture; UA(L)+CLP, low-dose ursolic acid + cecal ligation and puncture; UA(H)+CLP, high-dose ursolic acid + cecal ligation and puncture; BUN, blood urea nitrogen; Scr, serum creatinine; AKI, acute kidney injury. ${ }^{*} \mathrm{P}<0.05 ;{ }^{* * *} \mathrm{P}<0.001 ;{ }^{* * * *} \mathrm{P}<0.001$
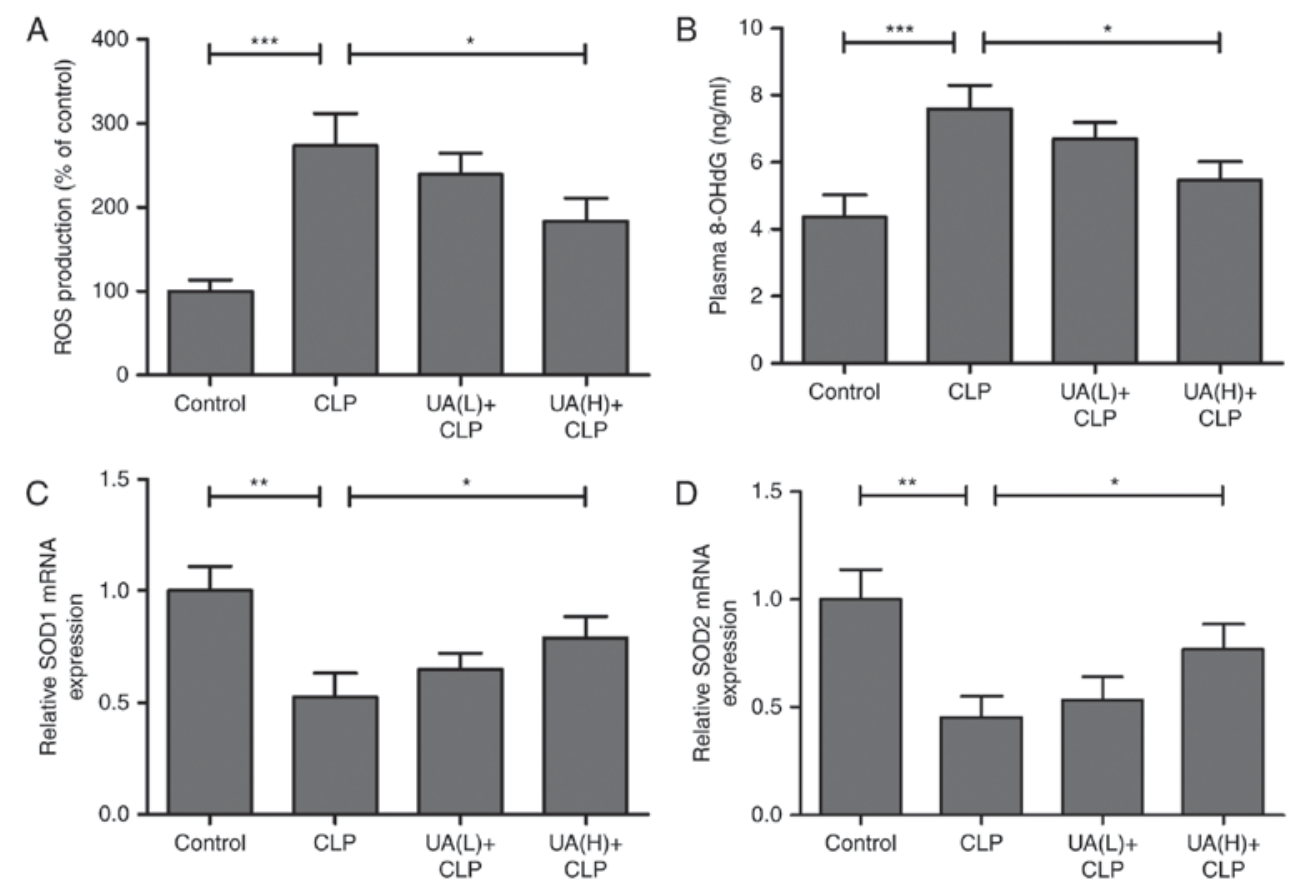

Figure 3. UA inhibits sepsis-induced oxidative stress. Renal tissues were harvested and homogenized in phosphate buffer at $24 \mathrm{~h}$ after CLP surgery in each group, and (A) ROS was measured using a luminometer. (B) Plasma levels of 8-OHdG were measured using a DNA damage ELISA kit. mRNA expression levels of (C) SOD1 and (D) SOD2 were measured by reverse transcription-quantitative polymerase chain reaction. ${ }^{*} \mathrm{P}<0.05 ;{ }^{* * *} \mathrm{P}<0.001 ;{ }^{* * * *} \mathrm{P}<0.001 . \mathrm{UA}$, ursolic acid; CLP, cecal ligation and puncture; ROS, reactive oxygen species; 8-OHdG, 8-hydroxy-2'-deoxyguanosine; SOD1, superoxide dismutase 1; SOD2, superoxide dismutase 2 .

demonstrated that treatment with high concentrations of UA attenuated the CLP-activated increase in TNF- $\alpha$, IL-1 $\beta$ and IL-6 expression $(\mathrm{P}<0.05)$. As shown in Fig. 5A, B and C, it was found that pretreatment with high concentrations of UA significantly suppressed the CLP-induced increase in TNF- $\alpha$, IL-1 $\beta$ and IL- 6 protein expression in kidney tissues from septic mice $(\mathrm{P}<0.01)$. Consequently, we examined whether the upstream NF- $\mathrm{\kappa B}$ signal transduction pathway was also involved in CLP-induced AKI. The transcription factor NF- $\kappa \mathrm{B}$ is considered to be the primary mediator of the inflammatory response (25), and the inhibition of NF- $\mathrm{KB}$ has been shown to alleviate sepsis-associated AKI in rats $(5,26)$. Therefore, the present study evaluated the effect of UA on the nuclear translocation of NF- $\kappa B$. The results demonstrated that the expression of NF- $\mathrm{KB}$ was significantly increased in the CLP group compared with control group $(\mathrm{P}<0.001)$. Conversely, 

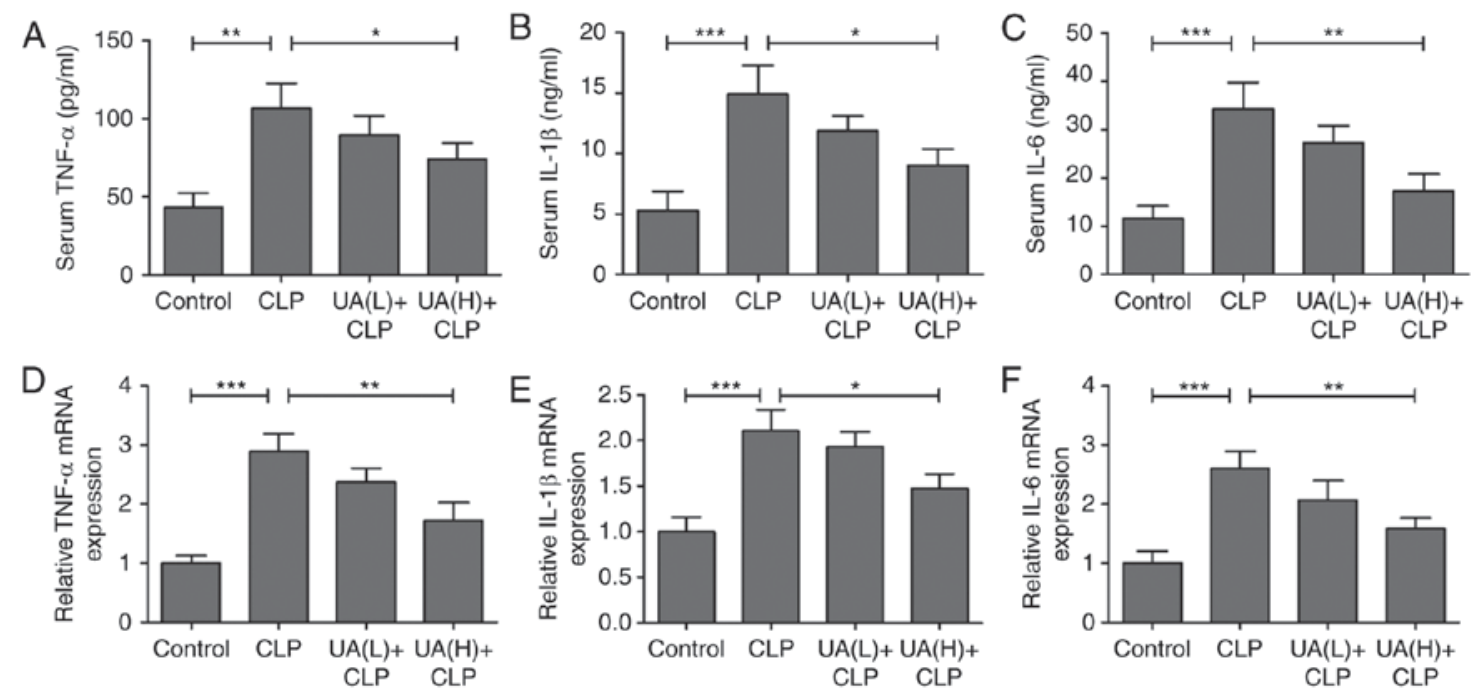

Figure 4. UA inhibits inflammatory cytokine levels in septic mice. Serum levels of (A) TNF- $\alpha$, (B) IL-1 $\beta$ and (C) IL-6 were detected using mouse bioactive ELISA assays in CLP-induced septic mice $(n=8)$. The mRNA expression levels of (D) TNF- $\alpha$, (E) IL-1 $\beta$ and (F) IL-6 were measured by reverse transcription-quantitative polymerase chain reaction $(\mathrm{n}=8) .{ }^{*} \mathrm{P}<0.05 ;{ }^{* *} \mathrm{P}<0.001 ;{ }^{* * * *} \mathrm{P}<0.001$. UA, ursolic acid; CLP, cecal ligation and puncture; TNF- $\alpha$, tumor necrosis factor- $\alpha$; IL-1 $\beta$, interleukin-1 $\beta$; IL-6, interleukin-6.

A
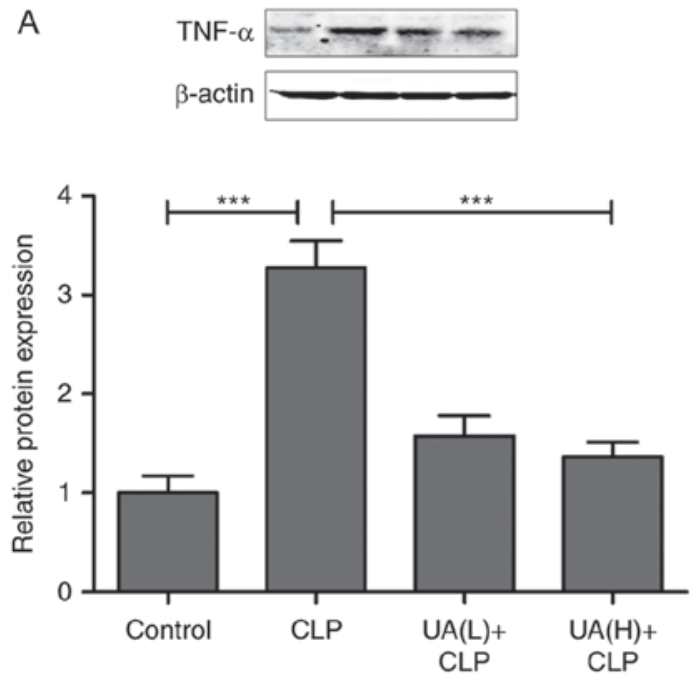

C
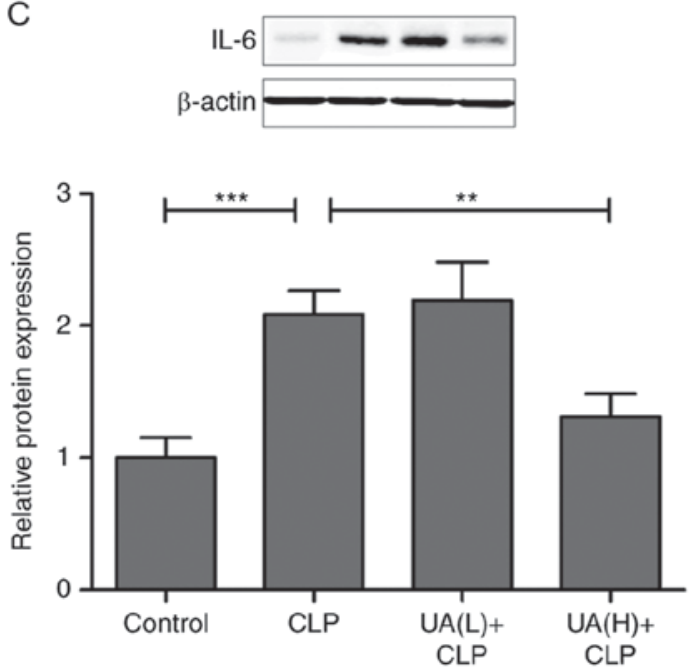

B
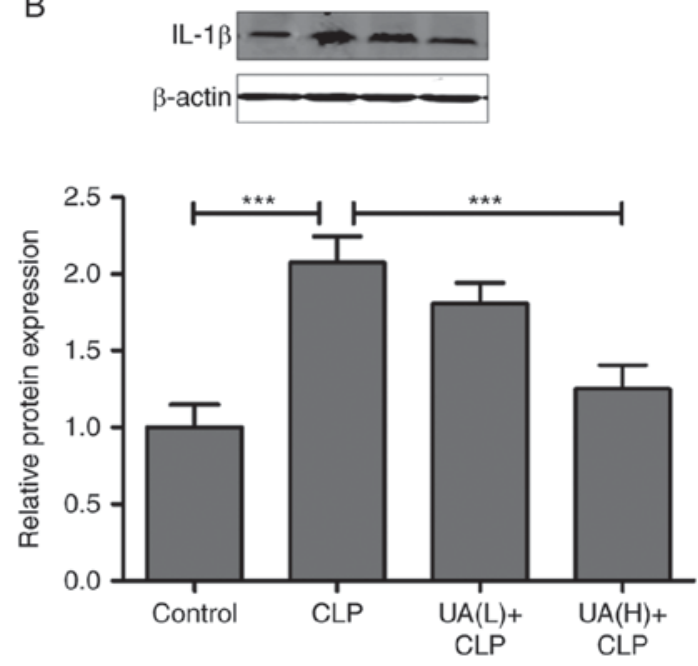

D
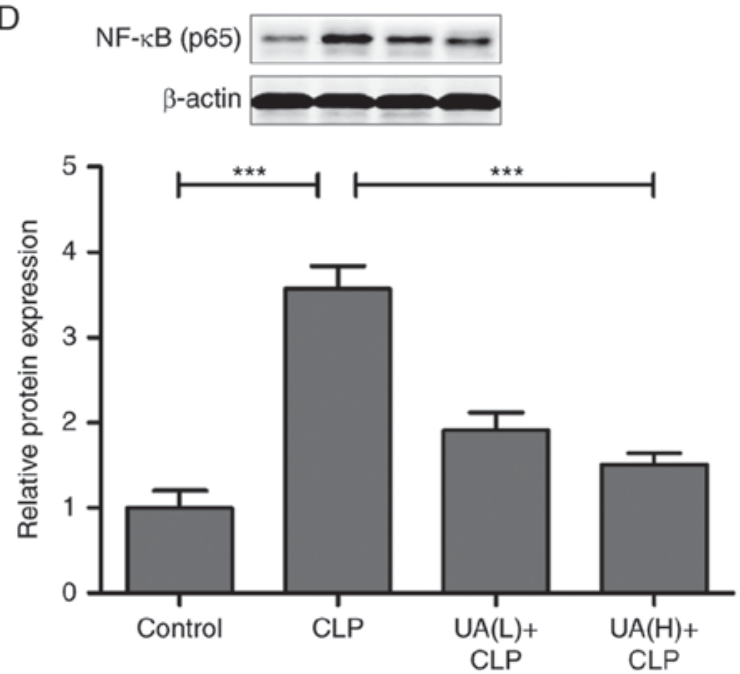

Figure 5. UA inhibits NF- $\kappa B$, TNF- $\alpha$, IL-1 $\beta$ and IL-6 protein expression in the kidney from septic mice. The levels of (A) TNF- $\alpha$, (B) IL-1 $\beta$, (C) IL-6 and (D) NF- $\mathrm{kB}$ protein expression were measured by western blotting and densitometric analysis in each group. ${ }^{*} \mathrm{P}<0.05 ;{ }^{* * *} \mathrm{P}<0.001$; ${ }^{* * * *} \mathrm{P}<0.001$. UA, ursolic acid; CLP, cecal ligation and puncture; TNF- $\alpha$, tumor necrosis factor- $\alpha$; IL-1 $\beta$, interleukin-1 $\beta$; IL-6, interleukin-6; NF- $\kappa B$, necrosis factor- $-\mathrm{kB}$. 
the administration of high concentrations of UA significantly reversed the increase in NF- $\kappa \mathrm{B}$ expression in the kidney from septic mice $(\mathrm{P}<0.001$; Fig. 5D).

\section{Discussion}

The present study used the experimental model of CLP-induced AKI to examine the effects of UA on sepsis-associated kidney injury in mice. First, results demonstrated that UA administration improved survival in septic mice induced by CLP surgery. The treatment of UA revealed protection against AKI induced by CLP surgery, including alleviation of glomerular damage and vacuolization in the proximal tubules. Furthermore, the effects of UA on oxidative stress and inflammation in septic mice were determined. The findings suggested that the high concentrations of UA $(20 \mathrm{mg} / \mathrm{kg})$ significantly protected against sepsis-induced AKI by inhibiting oxidative stress and inflammatory cytokines in the kidney from septic mice. It is well known that the concentration of UA is flexible in different experimental research. Consistent with previous reports, the dose of UA in rodent animal model is used at the same magnitude (16).

Mounting evidence has demonstrated that high free-radical concentrations are generated in patients with sepsis syndrome, and the balance between oxidation and anti-oxidation is markedly disturbed $(5,27)$. Increased production of ROS is one of the key features of sepsis, which may cause enhanced AKI $(5,21,28)$. In the present study, the findings revealed that ROS production was significantly increased by CLP surgery, whereas pretreatment with high concentrations of UA significantly inhibited CLP-induced ROS production in the kidney of septic mice, which suggested that UA protects against sepsis-induced AKI by inhibiting oxidative stress damage. A previous study indicated that SOD activity is significantly downregulated in septic patients, which is negatively associated with the severity of sepsis (29). In addition, the levels of the antioxidant SOD notably decreased in septic rats in every region of the brain (30). In the present study, it was found that the mRNA expression levels of SOD1 and SOD2 were significantly decreased in the kidney from septic mice, and high concentrations of UA treatment significantly reversed CLP-induced downregulation of SOD1 and SOD2. These results concur with previous observations that an imbalance exists between oxidants and antioxidants during sepsis (31). The findings of the present study demonstrated an anti-oxidant role for UA in septic mice.

TNF- $\alpha$, IL- $1 \beta$ and IL- 6 may be readily activated by pathological conditions, and are biomarkers of early inflammatory responses, as confirmed in previous studies (32-34). Under septic conditions, the expression of inflammatory cytokines, such as IL-1 $\beta$, IL- 6 and TNF- $\alpha$, are significantly upregulated in pathological tissues, including lung (17), cerebrum $(18,30)$ and kidney $(3,5)$. Clinical studies have found that the levels of TNF- $\alpha$ and IL- $1 \beta$ are increased in the serum of septic patients (35). Inhibition of TNF- $\alpha$ and IL-1 $\beta$ alleviates the progression of sepsis in animal models $(18,28)$. In the present study, it was demonstrated that serum levels of TNF- $\alpha$, IL-1 $\beta$ and IL- 6 and mRNA and protein expression levels of TNF- $\alpha$, IL-1 $\beta$ and IL- 6 in kidney were significantly increased in septic mice; however, pretreatment with UA attenuated the inflammatory response in septic mice, resulting in the downregulation of TNF- $\alpha$, IL- 6 and IL-1 $\beta$ levels in serum and kidney tissues. Inflammatory signals are known to merge in the activation of the $\mathrm{NF}-\kappa \mathrm{B}$ signaling pathway, and NF- $\kappa \mathrm{B}$ has been shown to serve a critical role in modulating mortality in experimental sepsis $(36,37)$. The results of the present study demonstrated that $\mathrm{NF}-\kappa \mathrm{B}$ was significantly increased in septic mice. Consistent with several original studies $(16,17)$, the findings of the present study also revealed that UA has anti-inflammatory properties by inhibiting $\mathrm{NF}-\kappa \mathrm{B}$ expression. Previous studies indicated that targeted knockout of IL-17A protects against sepsis-associated AKI and host defense $(38,39)$. The precise underlying pathogenic mechanism of TNF- $\alpha$, IL-1 $\beta$ or IL- 6 knockout in sepsis-induced AKI will be discussed in future studies. In addition, a more in-depth kinetic analysis of the inflammatory markers in septic mice is to be explored.

To the best of our knowledge, the present study provides evidence for the first time that UA is a novel therapeutic drug that protects against sepsis-induced AKI, and the underlying mechanism was mediated, at least partially, by inhibiting oxidative stress and inflammatory responses. The present study also provides a possible method for therapy sepsis-induced AKI in clinical practice. However, cross-sectional and longitudinal studies are required to determine the proper concentration of UA, which will be performed in future studies.

\section{Acknowledgements}

Not applicable.

\section{Funding}

No funding was received.

\section{Availability of data and materials}

The analyzed data sets generated during the study are available from the corresponding author on reasonable request.

\section{Authors' contributions}

Study design: ZW and ZZ; literature research, data acquisition and data analysis: ZZ, RC, ZW and HZ; manuscript preparation and manuscript editing: $\mathrm{ZZ}$ and $\mathrm{RC}$; manuscript review: $\mathrm{ZW}, \mathrm{ZZ}, \mathrm{HZ}$ and $\mathrm{RC}$; final approval of the version of the manuscript to be published: ZZ, HZ, RC and ZW; histological examination: RC; establishment of animal model: HZ.

\section{Ethics approval and consent to participate}

The animal study was approved by the Animal Care and Use Committee at the Beijing Tsinghua Changgung Hospital (Beijing, China; permit number: TCH-2015-0019).

\section{Consent for publication}

Not applicable.

\section{Competing interests}

The authors declare they have no competing interests. 


\section{References}

1. Zhang A, Cai Y, Wang PF, Qu JN, Luo ZC, Chen XD, Huang B, Liu Y, Huang WQ, Wu J and Yin YH: Diagnosis and prognosis of neutrophil gelatinase-associated lipocalin for acute kidney injury with sepsis: A systematic review and meta-analysis. Crit Care 20: 41, 2016.

2. Lopes JA, Fernandes P, Jorge S, Resina C, Santos C, Pereira A, Neves J, Antunes F and Gomes da Costa A: Long-term risk of mortality after acute kidney injury in patients with sepsis: A contemporary analysis. BMC Nephrol 11: 9, 2010.

3. Zarjou A and Agarwal A: Sepsis and acute kidney injury. J Am Soc Nephrol 22: 999-1006, 2011.

4. Murugan R, Karajala-Subramanyam V, Lee M, Yende S, Kong L, Carter M, Angus DC and Kellum JA; Genetic and Inflammatory Markers of Sepsis (GenIMS) Investigators: Acute kidney injury in non-severe pneumonia is associated with an increased immune response and lower survival. Kidney Int 77: 527-535, 2010.

5. Li N, Xie H, Li L, Wang J, Fang M, Yang N and Lin H: Effects of honokiol on sepsis-induced acute kidney injury in an experimental model of sepsis in rats. Inflammation 37: 1191-1199, 2014.

6. Quoilin C, Mouithys-Mickalad A, Lécart S, Fontaine-Aupart MP and Hoebeke M: Evidence of oxidative stress and mitochondrial respiratory chain dysfunction in an in vitro model of sepsis-induced kidney injury. Biochim Biophys Acta 1837: 1790-1800, 2014.

7. Weng TI, Wu HY, Kuo CW and Liu SH: Honokiol rescues sepsis-associated acute lung injury and lethality via the inhibition of oxidative stress and inflammation. Intensive Care Med 37: 533-541, 2011.

8. Jin H, Pi J, Yang F, Jiang J, Wang X, Bai H, Shao M, Huang L, Zhu H, Yang P, et al: Folate-chitosan nanoparticles loaded with ursolic acid confer anti-breast cancer activities in vitro and in vivo. Sci Rep 6: 30782, 2016.

9. Chu SM, Shih WT, Yang YH, Chen PC and Chu YH: Use of traditional Chinese medicine in patients with hyperlipidemia: A population-based study in Taiwan. J Ethnopharmacol 168 : 129-135, 2015

10. Guo X, Zhang L, Quan S, Hong Y, Sun L and Liu M: Isolation and identification of Triterpenoid compounds in the fruits of Chaenomeles lagenaria (Loisel.) Koidz. Zhongguo Zhong Yao Za Zhi 23: 546-547, 576, 1998 (In Chinese).

11. Shen H, Cheng T, Qiao C, Su Z and Li C: Antitumor effect in vitro and immuno-response in vivo of fructus Mume. Zhongguo Zhong Yao Za Zhi 20: 365-368, 1995 (In Chinese).

12. Alvarado HL, Abrego G, Garduño-Ramirez ML, Clares B, Calpena AC and García ML: Design and optimization of oleanolic/ursolic acid-loaded nanoplatforms for ocular anti-inflammatory applications. Nanomedicine 11: 521-530, 2015.

13. Ma JQ, Ding J, Zhang L and Liu CM: Ursolic acid protects mouse liver against $\mathrm{CCl} 4$-induced oxidative stress and inflammation by the MAPK/NF- $\mathrm{B}$ pathway. Environ Toxicol Pharmacol 37: 975-983, 2014.

14. Prasad S, Yadav VR, Sung B, Gupta SC, Tyagi AK and Aggarwal BB: Ursolic acid inhibits the growth of human pancreatic cancer and enhances the antitumor potential of gemcitabine in an orthotopic mouse model through suppression of the inflammatory microenvironment. Oncotarget 7: 13182-13196, 2016.

15. Wang ZH, Hsu CC, Huang CN and Yin MC: Anti-glycative effects of oleanolic acid and ursolic acid in kidney of diabetic mice. Eur J Pharmacol 628: 255-260, 2010.

16. Ma JQ, Ding J, Xiao ZH and Liu CM: Ursolic acid ameliorates carbon tetrachloride-induced oxidative DNA damage and inflammation in mouse kidney by inhibiting the STAT3 and NF- $\kappa$ B activities. Int Immunopharmacol 21: 389-395, 2014.

17. Hu Z, Gu Z, Sun M, Zhang K, Gao P, Yang Q and Yuan Y: Ursolic acid improves survival and attenuates lung injury in septic rats induced by cecal ligation and puncture. J Surg Res 194: 528-536, 2015.

18. Chen X, Wan Y, Zhou T, Li J and Wei Y: Ursolic acid attenuates lipopolysaccharide-induced acute lung injury in a mouse model. Immunotherapy 5: 39-47, 2013

19. Oh SJ, Kim JH and Chung DH: NOD2-mediated suppression of CD55 on neutrophils enhances C5a generation during polymicrobial sepsis. PLoS Pathog 9: e1003351, 2013.

20. Livak KJ and Schmittgen TD: Analysis of relative gene expression data using real time quantitative PCR and the 2(-Delta Delta C(T)) method. Methods 25: 402-408, 2001.
21. Wang P, Huang J, Li Y, Chang R, Wu H, Lin J and Huang Z: Exogenous carbon monoxide decreases sepsis-induced acute kidney injury and inhibits NLRP3 inflammasome activation in rats. Int J Mol Sci 16: 20595-20608, 2015.

22. Johns EJ, O'Shaughnessy B, O'Neill S, Lane B and Healy V. Impact of elevated dietary sodium intake on $\mathrm{NAD}(\mathrm{P}) \mathrm{H}$ oxidase and SOD in the cortex and medulla of the rat kidney. Am J Physiol Regul Integr Comp Physiol 299: R234-R240, 2010.

23. Li X, Wang X, Zheng M and Luan QX: Mitochondrial reactive oxygen species mediate the lipopolysaccharide-induced pro-inflammatory response in human gingival fibroblasts. Exp Cell Res 347: 212-221, 2016.

24. Closa D and Folch-Puy E: Oxygen free radicals and the systemic inflammatory response. IUBMB Life 56: 185-191, 2004.

25. Leslie KL, Song GJ, Barrick S, Wehbi VL, Vilardaga JP, Bauer PM and Bisello A: Ezrin-radixin-moesin-binding phosphoprotein 50 (EBP50) and nuclear factor- $\kappa \mathrm{B}$ (NF- $\kappa \mathrm{B})$ : A feed-forward loop for systemic and vascular inflammation. J Biol Chem 288: 36426-36436, 2013.

26. Souza AC, Volpini RA, Shimizu MH, Sanches TR, Camara NO, Semedo P, Rodrigues CE, Seguro AC and Andrade L: Erythropoietin prevents sepsis-related acute kidney injury in rats by inhibiting NF- $\kappa \mathrm{B}$ and upregulating endothelial nitric oxide synthase. Am J Physiol Renal Physiol 302: F1045-F1054, 2012.

27. Galley HF, Davies MJ and Webster NR: Xanthine oxidase activity and free radical generation in patients with sepsis syndrome. Crit Care Med 24: 1649-1653, 1996.

28. Zhao H, Liu Z, Shen H, Jin S and Zhang S: Glycyrrhizic acid pretreatment prevents sepsis-induced acute kidney injury via suppressing inflammation, apoptosis and oxidative stress. Eur J Pharmacol 781: 92-99, 2016.

29. Yao L, Liu Z, Zhu J, Li B, Chai C and Tian Y: Clinical evaluation of circulating microRNA-25 level change in sepsis and its potential relationship with oxidative stress. Int J Clin Exp Pathol 8: 7675-7684, 2015.

30. Chen Q, Yu W, Shi J, Shen J, Gao T, Zhang J, Xi F, Li J and Li N: Insulin alleviates the inflammatory response and oxidative stress injury in cerebral tissues in septic rats. J Inflamm (Lond) 11: 18 , 2014.

31. He L, Peng X, Zhu J, Chen X, Liu H, Tang C, Dong Z, Liu F and Peng Y: Mangiferin attenuate sepsis-induced acute kidney injury via antioxidant and anti-inflammatory effects. Am J Nephrol 40: 441-450, 2014

32. Sedlár M, Kudrnová Z, Erhart D, Trca S, Kvasnicka J, Krska Z, Mazoch J, Malíková I, Zeman M and Linhart A: Older age and type of surgery predict the early inflammatory response to hip trauma mediated by interleukin-6 (IL-6). Arch Gerontol Geriatr 51: e1-e6, 2010.

33. Canault M, Peiretti F, Mueller C, Kopp F, Morange P, Rihs S, Portugal H, Juhan-Vague I and Nalbone G: Exclusive expression of transmembrane TNF-alpha in mice reduces the inflammatory response in early lipid lesions of aortic sinus. Atherosclerosis 172: 211-218, 2004

34. Kajahn J, Franz S, Rueckert E, Forstreuter I, Hintze V, Moeller S and Simon JC: Artificial extracellular matrices composed of collagen I and high sulfated hyaluronan modulate monocyte to macrophage differentiation under conditions of sterile inflammation. Biomatter 2: 226-236, 2012.

35. Moldawer LL: Interleukin-1, TNF alpha and their naturally occurring antagonists in sepsis. Blood Purif 11: 128-133, 1993

36. Liu Z, Wang Y, Ning Q, Gong C, Zhang Y, Zhang L, Bu X and Jing G: The role of spleen in the treatment of experimental lipopolysaccharide-induced sepsis with dexmedetomidine. Springerplus 4: 800, 2015.

37. Wu H, Liu J, Li W, Liu G and Li Z: LncRNA-HOTAIR promotes TNF- $\alpha$ production in cardiomyocytes of LPS-induced sepsis mice by activating NF- $\kappa$ B pathway. Biochem Biophys Res Commun 471: 240-246, 2016

38. Luo CJ, Luo F, Zhang L, Xu Y, Cai GY, Fu B, Feng Z, Sun XF and Chen XM: Knockout of interleukin-17A protects against sepsis-associated acute kidney injury. Ann Intensive Care 6: 56, 2016.

39. Ogiku M,Kono H,Hara M,Tsuchiya M and Fujii H: Interleukin-17A plays a pivotal role in polymicrobial sepsis according to studies using IL-17A knockout mice. J Surg Res 174: 142-149, 2012.

This work is licensed under a Creative Commons Attribution-NonCommercial-NoDerivatives 4.0 International (CC BY-NC-ND 4.0) License. 